\title{
Cortical Auditory Evoked Potentials in Response to Frequency Changes with Varied Magnitude, Rate, and Direction
}

\author{
Bernard M.D. Vonck, ${ }^{1,2}$ Marc J.W. Lammers, ${ }^{1,2,3}$ Marjoljun van der WaAls, ${ }^{1}$ \\ Gijsbert A. van Zanten, ${ }^{1,2}$ and Huib Versnel ${ }^{1,2}$ (D) \\ ${ }^{1}$ Department of Otorhinolaryngology and Head $\mathcal{E}$ Neck Surgery, University Medical Center Utrecht, Room G.02.531, \\ P.O. Box 855003508 GA, Utrecht, The Netherlands \\ ${ }^{2}$ UMC Utrecht Brain Center, Utrecht, The Netherlands \\ ${ }^{3}$ BC Rotary Hearing and Balance Centre at St. Paul's Hospital, University of British Columbia, Vancouver, British Columbia, \\ Canada
}

Received: 11 June 2018; Accepted: 20 May 2019; Online publication: 5 June 2019

\begin{abstract}
Recent literature on cortical auditory evoked potentials has focused on correlations with hearing performance with the aim to develop an objective clinical tool. However, cortical responses depend on the type of stimulus and choice of stimulus parameters. This study investigates cortical auditory evoked potentials to sound changes, so-called acoustic change complexes (ACC), and the effects of varying three stimulus parameters. In twelve normal-hearing subjects, ACC waveforms were evoked by presenting frequency changes with varying magnitude, rate, and direction. The N1 amplitude and latency were strongly affected by magnitude, which is known from the literature. Importantly, both of these N1 variables were also significantly affected by both rate and direction of the frequency change. Larger and earlier N1 peaks were evoked by increasing the magnitude and rate of the frequency change and with downward rather than upward direction of the frequency change. The P2 amplitude increased with magnitude and depended, to a lesser extent, on rate of the frequency change while direction had no effect on this peak. The N1-P2 interval was not affected by any of the stimulus parameters. In conclusion, the ACC is most strongly affected by magnitude and also substantially by rate and direction of the change. These stimulus dependencies should be consid-
\end{abstract}

Correspondence to: Huib Versnel · Department of Otorhinolaryngology and Head \& Neck Surgery · University Medical Center Utrecht . Room G.02.531, P.O. Box 855003508 GA, Utrecht, The Netherlands. Telephone: +31(0)887557724; email: h.versnel@ umcutrecht.nl ered in choosing stimuli for ACCs as objective clinical measure of hearing performance.

Keywords: acoustic change complex, cortical auditory evoked potential, frequency modulation, electroencephalography

Abbreviations: ACC - Acoustic change complex; FM Frequency modulation; oct-Octave; RM-Repeated measures

\section{INTRODUCTION}

The ability of our auditory system to detect modulations within ongoing sounds is essential for daily life. It enables us to detect changes in our environment, to identify vowels and consonants, and also to appreciate pitch differences in musical compositions. Normal hearing listeners can identify very small frequency changes of less than $1 \%$ of the base frequency (Sek and Moore 1995; Amitay et al. 2006; Papakonstantinou et al. 2011). In the case of sensorineural hearing loss, frequency resolution capabilities become impaired, resulting in poorer speech understanding in especially noisy environments (Dreschler and Plomp 1985; Horst 1987; Noordhoek et al. 2001; Strelcyk and Dau 2009; Papakonstantinou et al. 2011). Psychophysical experiments revealed that patients with sensorineural hearing loss had frequency discrimination thresholds which correlated well with their speech perception in noise abilities (Noordhoek et al. 2001; Papakonstantinou et al. 2011). These findings indicate the importance of frequency discrimination in oral communication. 
The underlying neurophysiological alterations in response to frequency changes have been investigated using cortical auditory evoked potentials (Arlinger et al. 1976; McCandless and Rose 1970; Dimitrijevic et al. 2008; Harris et al. 2008; Pratt et al. 2009). The obligatory cortical auditory evoked potential is thought to mainly reflect neuronal activation in various primary and secondary auditory cortical fields (Eggermont and Ponton 2002; Martin et al. 2007, 2008). The response evoked by a change in a continuous stimulus is often called the acoustic change complex (ACC). This response can be elicited in response to changes within speech stimuli (Ostroff et al. 1998; Martin and Boothroyd 2000; Tremblay et al. 2003; Friesen and Tremblay 2006) and to intensity or frequency changes within continuous tones (McCandless and Rose 1970; Arlinger et al. 1976; Harris et al. 2007, 2008; Dimitrijevic et al. 2008; Pratt et al. 2009; Presacco and Middlebrooks 2018).

Recent literature has shown interest in the ACC and investigated its possible clinical applications (He et al. 2012; Brown et al. 2015, 2017; Chen and Small 2015; Kim 2015). The ACC has been reported to correlate with psychophysical measures in normal hearing adult subjects (He et al. 2012; Brown et al. 2017), and moreover, the ACC has been reliably evoked in various types of subjects such as young children, hearing aid users, cochlear implant users, and sedated cats (Tremblay et al. 2006; He et al. 2012; Brown et al. 2015; Chen and Small 2015; Presacco and Middlebrooks 2018). The ACC might therefore possess characteristics, which can be used for objective auditory assessment. Although recent research has aimed at the correlation of ACC measures (such as amplitude and latency) to psychophysical outcomes, these measures also depend on the choice of stimulus parameters. Knowledge on how different factors of frequency change stimuli affect ACC parameters is therefore essential for researchers investigating the ACC. Previous studies have revealed that ACCs elicited with frequency changes show larger amplitudes with increasing magnitude of the frequency change (McCandless and Rose 1970; Martin and Boothroyd 2000; Harris et al. 2008; Pratt et al. 2009; He et al. 2012). Harris et al. (2008) demonstrated that ACCs can even be evoked in response to small changes of less than $1 \%$ of the base frequency, closely resembling behavioral just noticeable frequency discrimination results. Besides the magnitude of the change, there are multiple parameters to choose for an acoustic change stimulus, see for instance, the differences in stimuli between abovementioned studies. The majority of studies using a pure tone stimulus, followed by a frequency change, studied only one direction of frequency change (frequency increase or decrease). With respect to rate of the frequency change, some authors did not report rates (Pratt et al. 2009; Brown et al. 2017) while others reported a constant duration of the change with varying magnitudes, thus resulting in varying velocities
(Dimitrijevic et al. 2008; Harris et al. 2008; Presacco and Middlebrooks 2018). Thus, since evidence is lacking on the effect of rate and direction, we aim to investigate the extent to which suprathreshold ACC is influenced by rate and direction, next to magnitude. This may help researchers and clinicians to determine stimulus parameters when recording ACCs, for example, the rate and/or direction of change that generates the clearest response or the steepest amplitude-change slope. This may attribute to development of the ACC into a clinically applicable objective measurement of auditory performance. Therefore, in the current study, we systematically varied magnitude, rate, and direction to elicit the ACC in young, normal-hearing subjects.

\section{METHODS}

\section{Subjects}

Twelve healthy, normal hearing volunteers, aged between 18 and 30 years old, agreed to participate in this study. Eleven participants were right handed, and one was left handed. Harris et al. (2008) reported that in a group of normal hearing subjects of this age, small frequency changes up to the threshold of around $0.8-1.8 \%$ frequency change of the base frequency elicited a cortical response. In older adults, aged 65-80 years, this threshold ranged from 1.2 to $3.4 \%$ of the base frequency (Harris et al. 2008). This study only included young subjects to diminish the possible effect of age on the ACC. Hearing thresholds of each participant measured by standard pure tone audiometry prior to the experiments were $<20 \mathrm{~dB} H \mathrm{HL}$ at each frequency $(0.25-8 \mathrm{kHz})$. None of the subjects reported a history of hearing loss or tinnitus.

\section{Stimuli and Recording Procedure}

Acoustic change complexes were evoked using tonal stimuli of $3300 \mathrm{~ms}$, consisting of three components (Fig. 1a): (a) a reference tone of $1000 \mathrm{~Hz}$ with a duration of approximately $3 \mathrm{~s}$, (b) a logarithmic frequency modulation (FM) sweep with a frequency change $\Delta f$, and (c) a 300-ms target tone with a frequency $1000-\Delta f$ or $1000+\Delta f \mathrm{~Hz}$. The FM sweep was generated using the following equation:

$$
y=\sin \left[\frac{2 \pi f_{0}}{R \log (2)} e^{R t \log (2)}-\varphi \cdot\right]
$$

with $y$ : FM sweep signal; $f_{0}$ : reference frequency in $\mathrm{Hz}$; $R$ : rate in octave per second; $t$ : time in seconds; and $\varphi$ : phase correction to start FM sweep at the final phase of the reference tone. 

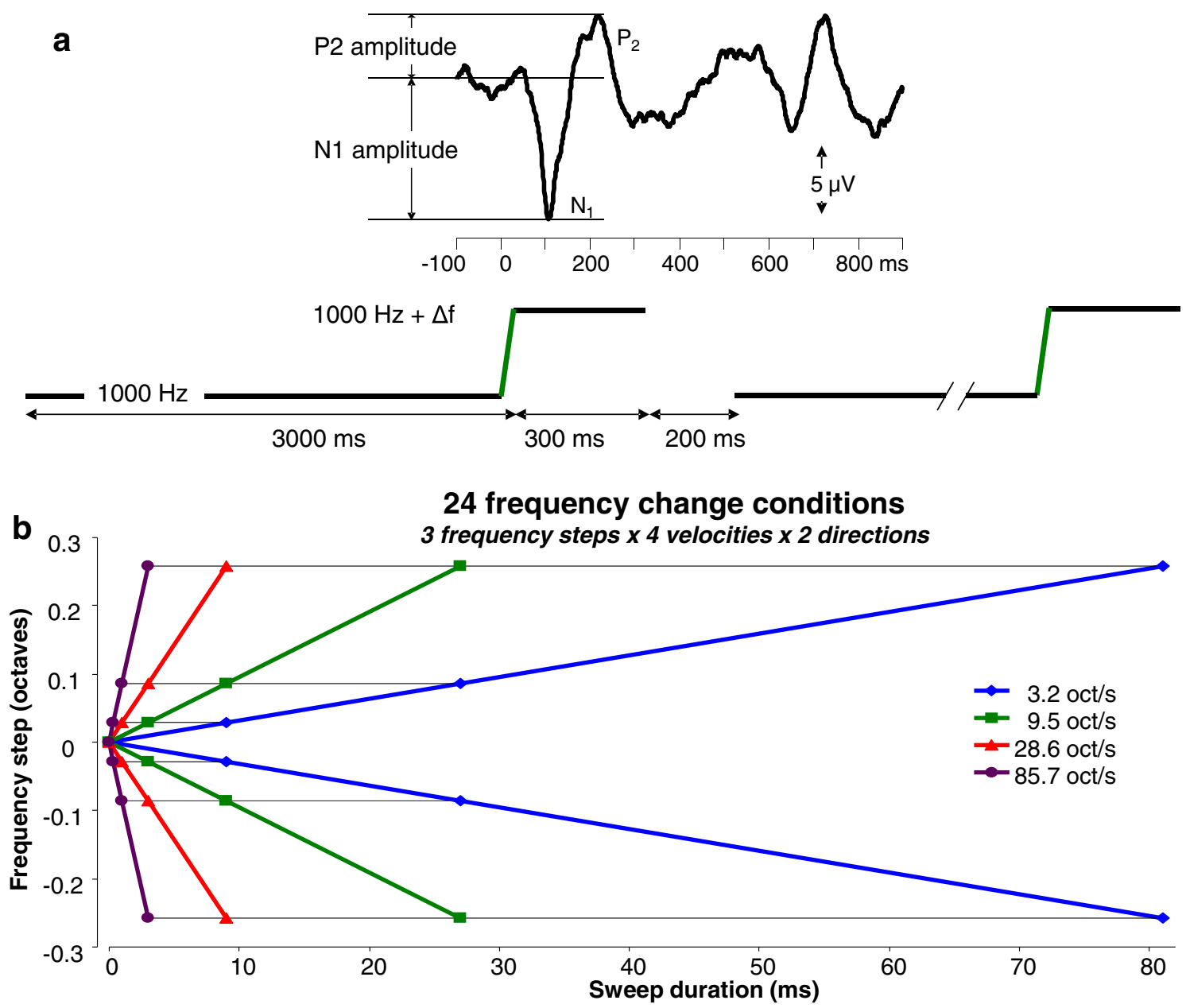

Fig. 1. a Schematic presentation of the stimulus. Stimuli consisted of 3 parts: (1) a $1000-\mathrm{Hz}$ pure tone with a duration of approximately $3 \mathrm{~s}$, which transfers after 2919 to $2999.7 \mathrm{~ms}$ into (2) an upward or downward frequency modulated sweep with a duration of 0.33 to $81 \mathrm{~ms}$ and then proceeds as a (3) 300-ms continuous tone with a frequency of $1000 \mathrm{~Hz} \pm \Delta f$. An inter stimulus interval of $200 \mathrm{~ms}$ was provided before the onset of the next repeated stimulus. The top graph demonstrates the corresponding acoustic change complex occurring at approximately $100 \mathrm{~ms}$ after the sweep and the cortical auditory evoked response at approximately $600 \mathrm{~ms}$ elicited by the

The silent interval between stimuli was $200 \mathrm{~ms}$. The choice of the relatively long duration of the reference tone was based on pilot data we obtained in 3 subjects, in which ACC amplitudes following a 3-s reference tone were substantially larger (by a factor $\sim 1.5$ ) than those following a 1-s reference tone. Time 0 of the recordings is defined by the onset of the frequency change.

Frequency changes of 3 different magnitudes were used: 0.028 oct (20 Hz for up change), 0.086, and 0.257 oct. The smallest magnitude of 0.028 oct corresponds to a $2 \%$ frequency change from the reference tone, which is expected to elicit an ACC since it is above reported thresholds $(0.8-1.8 \%)$ in young normal-hearing individuals (Harris et al. 2008). These 3 magnitudes were presented at 4 different FM onset of the new stimulus. b Schematic overview of all 24 stimulus conditions showing frequency step magnitude as a function of duration of the FM sweep. Downward frequency changes are indicated with negative values. Purple lines indicate the fastest sweeps of $85 \mathrm{oct} / \mathrm{s}$, the red line sweeps of $28 \mathrm{oct} / \mathrm{s}$, the green lines of $9.5 \mathrm{oct} / \mathrm{s}$, and the blue lines of $3.2 \mathrm{oct} / \mathrm{s}$. For each rate, frequency changes of $0.028,0.086$, and 0.257 oct in both upward and downward directions were used. The 24 frequency changes had varying durations: $0.33,1,3,9,27$, or $81 \mathrm{~ms}$.

sweep velocities, 3.2, 9.5, 28, and 85 oct/s, which generated 12 stimulus conditions. These 12 frequency changes were presented in both the upward $(+\Delta f)$ and downward $(-\Delta f)$ directions, resulting in a total of 24 different frequency change conditions for each participant (Fig. 1b). According to the combinations of steps and velocities, the 24 frequency changes had durations of $0.33,1,3,9,27$, or $81 \mathrm{~ms}$, as illustrated in Fig. $1 b$. The exact duration of the reference tone was $3000 \mathrm{~ms}$ minus the duration of the change and thus varied between 2919 and $2999.7 \mathrm{~ms}$. Sound stimuli were generated using MATLAB (version 7.11.0, Mathworks, Natick, MA, USA) at a sample frequency of $50 \mathrm{kHz}$ and presented monaurally to the left ear through a TDH-39 headphone at a level of $75 \mathrm{~dB}$ SPL. 
All 24 conditions were presented in a random order for each participant.

Participants were seated in a comfortable reclining chair in an electrically shielded, sound attenuated booth, and were allowed to watch a silent, captioned movie. They were carefully instructed prior to each recording to minimize movements and to fixate on the center of the video screen to minimize muscle and eye movement artifacts. Electrophysiological responses were recorded by $\mathrm{Ag} / \mathrm{AgCl}$ electrodes placed according to the 10-20 system using a Medelec Synergy T-10 Evoked Potential system. The active electrode was placed at the vertex of the skull, $\mathrm{Cz}$; the contralateral mastoid (right) was used as reference electrode; and the ground electrode was placed on the forehead. Eye movements and blinks were monitored using electrodes above and below the eye, contralateral of the stimulated ear. Electrode impedances were kept below $5 \mathrm{k} \Omega$. The electrode signals were filtered from 0.01 to $100 \mathrm{~Hz}$ and recorded with a sampling rate of $50 \mathrm{kHz}$. Responses were acquired in a 1000-ms time window, including a prestimulus period of $100 \mathrm{~ms}$. Responses containing amplitudes of > $100 \mu \mathrm{V}$ at any electrode were rejected and excluded from the averaged response. For each condition, 100 accepted sweeps were averaged.

\section{Data Analyses}

Averaged evoked potential data were used for determining peak amplitudes and latencies for each subject. The first peak, P1, was considerably smaller compared to the following N1 and P2 peaks. The low signal-to-noise ratio of this peak impedes reliable determination of $\mathrm{P} 1$ amplitude and latency. Therefore, only the N1 and P2 were further analyzed. The N1 of the ACC was defined as the most negative peak at 70 to $170 \mathrm{~ms}$ after the onset of the frequency change. P2 was defined as the first pronounced positive peak occurring after N1 at 150 to $250 \mathrm{~ms}$ after the change. The N1 and P2 amplitudes were computed relative to baseline, which was assessed by the average prestimulus segment $(-100$ to $0 \mathrm{~ms}$; see Fig. 1a). The N1 latency was measured at the peak relative to stimulus onset, and the P2 latency was analyzed relative to the N1 latency. Statistical analyses were completed using SPSS version 22.0 software (IBM, Armonk, NY, USA). For each of the 24 conditions, amplitudes and latencies of both the N1 and P2 peaks were averaged across all 12 subjects. Repeated measures (rm) ANOVA was applied to evaluate differences between stimulus conditions with respect to the three within-subject factors magnitude (3 levels), rate (4 levels), and direction (2 levels). If the assumption of sphericity was violated, the Greenhouse-Geisser correction was applied; $p<0.05$ was considered significant for main effects and interactions. In a secondary evaluation, an effect of direction was analyzed by computing a direction selectivity index for amplitude: DSI = (Amplitude down - Amplitude up) / (Amplitude down + Amplitude up), as described e.g. by Nelken and Versnel (2000), and by computing latency differences between directions. DSI and latency differences were statistically tested using Bonferroni corrected $t$ tests.

\section{RESULTS}

Reproducible and clear ACC responses exhibiting the typical N1-P2 waveform morphology were evoked in all 12 subjects, for all 24 stimulus conditions. This is illustrated by Fig. 2, which shows the unfiltered responses evoked in one individual subject for the 24 different conditions. ACC responses with an N1 peak at approximately $100 \mathrm{~ms}$ are seen for all stimulus conditions. Around $600 \mathrm{~ms}$, a smaller N1-P2 waveform occurs elicited by the onset of the next repeated stimulus, which starts with the $1000 \mathrm{~Hz}$ reference tone prior to the frequency change as illustrated in Fig. 1a. In some recordings, a small N1-P2 can be identified at approximately $400 \mathrm{~ms}$, elicited in response to the offset of the stimulus.

Effects of Frequency Change Magnitude, Rate, and Direction on Amplitude

As is seen in the examples presented in Fig. 2, the ACC amplitude increased with increasing magnitude of the frequency change. In addition, the ACC amplitude increased with increasing rate. This effect can be well observed within the top traces of Fig. 2 (ACCs evoked by the largest frequency change of $0.257 \mathrm{oct}$ ). With respect to direction, there appears to be little difference in ACC amplitude between downward (left traces) and upward (right traces) sweeps in this subject. Figure 3a depicts the averaged amplitudes of all subjects $(N=12)$ as a function of frequency change magnitude and shows that the N1 amplitude of the ACC was significantly affected by magnitude, rate, and direction of the frequency step. Amplitudes increased with increasing magnitude of the frequency changes (rm ANOVA; $F(2,22)=50.0, p$ $<0.0001)$. The largest increase in amplitude occurred with the increase from 0.028 to 0.086 oct. Furthermore, the $\mathrm{N} 1$ amplitude increased with an increase in rate $(\mathrm{rm}$ ANOVA; $F(3,33)=11.1, p<0.0001)$. Amplitudes evoked by downward changes in frequency were larger compared to amplitudes evoked by upward changes (rm ANOVA; $F(1,11)=9.9, p=0.009)$. Interaction effects were revealed between magnitude and rate ( $\mathrm{rm}$ ANOVA; magnitude $\times$ rate $F(3,33)=3.1, p=0.038)$ and between 


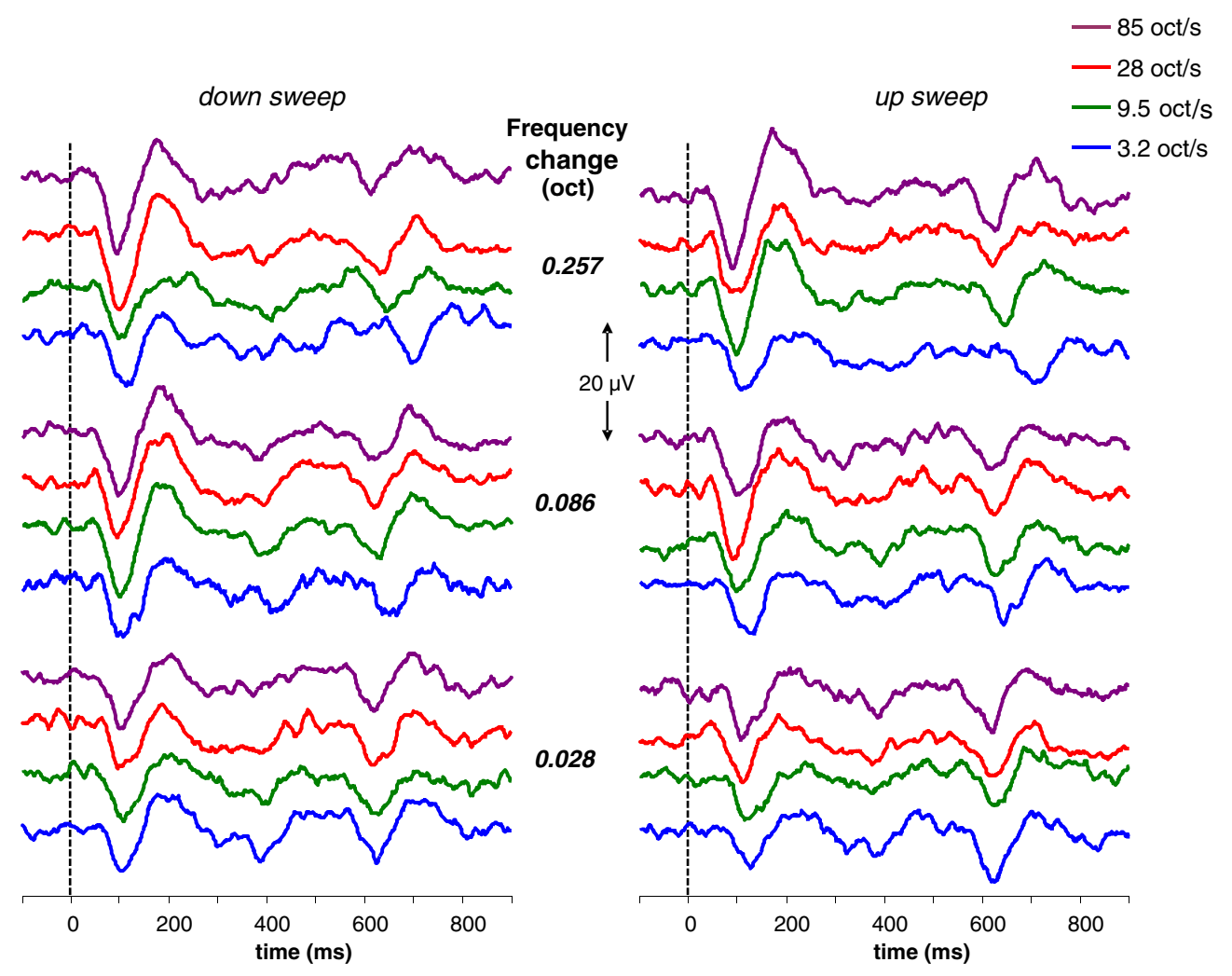

Fig. 2. Individual and unfiltered recordings obtained in one subject displaying the acoustic change responses for all 24 conditions separately. The frequency change starts at $0 \mathrm{~ms}$, and the acoustic change complex can be identified in all traces at approximately $100 \mathrm{~ms}$ after the frequency change. The response occurring around $600 \mathrm{~ms}$ after the frequency change is the cortical onset response on the next repeated stimulus. The eight top traces demonstrate the

ACCs obtained with the largest frequency change, i.e., of 0.257 oct; the middle traces the responses for a frequency change of 0.086 oct; and the bottom traces for a change of 0.028 oct. Purple traces indicate the ACCs for the fastest sweeps, i.e., of $85 \mathrm{oct} / \mathrm{s}$, the red traces for $28 \mathrm{oct} / \mathrm{s}$, the green traces for $9.5 \mathrm{oct} / \mathrm{s}$, and the blue traces for $3.2 \mathrm{oct} / \mathrm{s}$. The traces on the left show ACCs for downward and the traces on the right for upward frequency changes

rate and direction ( $\mathrm{rm}$ ANOVA; rate $\times$ direction $F(6,66)=3.1, p=0.011)$. Figure 3 illustrates these effects: a small amplitude difference between velocities at the smallest frequency change in contrast to a larger difference at the largest frequency change and a larger rate effect for downward than for upward changes.

As with the N1 component, the P2 increased with magnitude (rm ANOVA; $F(2,22)=30.2, p<0.0001)$ and, to a lesser extent, with rate of the frequency change ( $\mathrm{rm}$ ANOVA $F(3,33)=3.1, p=0.039)$ (Fig. 3b). In contrast to the N1 peak, P2 amplitude was not affected by direction. An interaction effect for the P2 amplitude was revealed between magnitude and rate of the frequency change ( $r$ ANOVA; magnitude $\times$ rate $F(6,66)=3.5, p=0.005)$. This effect was reflected by a larger increase in amplitudes for higher than for lower velocities, in especially the upward sweeps (Fig. 3b). With upward frequency changes of 0.028 and 0.086 oct, the slower sweeps showed larger amplitudes while with a frequency change of 0.257 the faster sweeps showed larger amplitudes. P2 amplitude was not affected by direction of the change ( $r m$ ANOVA; $F(2,22)=0.01, p=0.924)$.

Figure 4 shows the N1 amplitude as function of the logarithm of the sweep duration for each of the four rates. Considering the goodness of the fit $\left(R^{2}>0.8\right)$, it appears that the increase of the $\mathrm{N} 1$ amplitude, at least in the range applied here, can be described as a logarithmic function of the sweep duration. The slope varied from $3.1 \mu \mathrm{V}$ for a rate of $3.2 \mathrm{oct} / \mathrm{s}$ to $4.8 \mu \mathrm{V}$ for a rate of 85 oct/s. The shallower slope for slower changes reflects the interaction between magnitude and rate. Since the magnitude of change is proportional to the sweep duration (magnitude $=$ rate $\times$ duration), the N1 amplitude increases logarithmically with the magnitude.

\section{Effects of Frequency Change Magnitude, Rate, and Direction on Latency}

The N1 latency was significantly affected by magnitude, rate and direction of the frequency change (Fig. 5). Latencies decreased with an increase in magnitude of the frequency change ( $\mathrm{rm}$ ANOVA; $F(2,22)=86.2, p<0.0001)$ and as with the N1 ampli- 

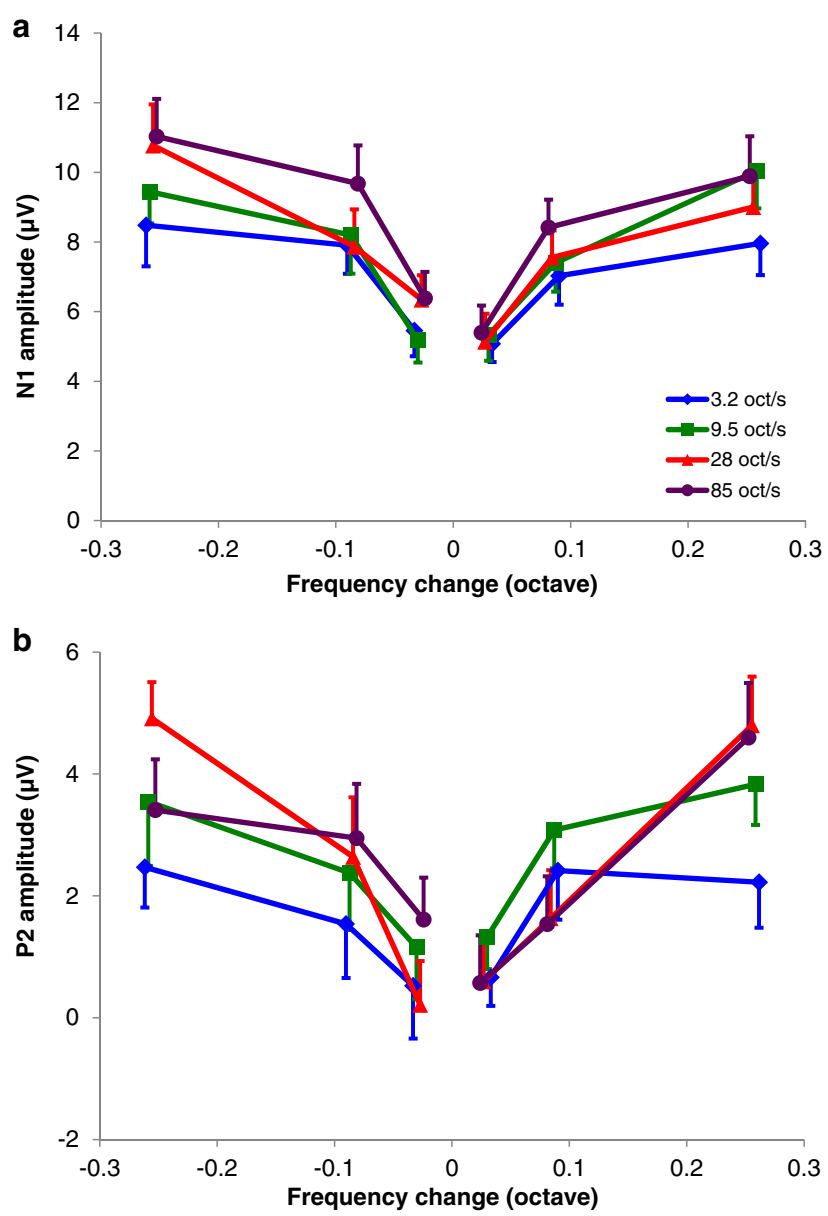

Fig. 3. Average $\mathrm{N} 1$ (a) and P2 (b) amplitudes as a function of magnitude of the frequency change $(n=12)$. Downward frequency changes are presented on the left (indicated with negative values) and upward changes on the right. Purple lines indicate the sweeps of $85 \mathrm{oct} / \mathrm{s}$, red lines of $28 \mathrm{oct} / \mathrm{s}$, green lines of $9.5 \mathrm{oct} / \mathrm{s}$, and the blue lines of $3.2 \mathrm{oct} / \mathrm{s}$. Error bars indicate standard errors of the mean

tude, this effect was strongest with the increase from 0.028 to 0.086 oct. Furthermore, the latency was affected by rate of the change $(F(3,33)=40.6$, $p<0.0001$ ), i.e., latency decreased with increasing rate. In particular, the slowest rate of 3.2 oct $/ \mathrm{s}$ produced substantially longer N1 latencies than the other velocities, by approximately $10 \mathrm{~ms}$, over all frequency changes (Fig. 5). In addition, for the largest frequency change of 0.257 oct, the N1 latency was longer for both the slowest velocities compared to the two highest velocities. This was confirmed by interaction effects between magnitude and rate of the frequency change $(\mathrm{rm}$ ANOVA; magnitude $\times$ rate $F(6,66)=6.45, p<0.0001)$ and between direction, magnitude, and rate of the frequency change ( $\mathrm{rm}$ ANOVA; direction $\times$ magnitude $\times$ rate $F(6,66)=2.41$, $p=0.036)$. As shown in Fig. 5, the average N1 latencies per stimulus condition were shorter when evoked in response to downward changes (range of averages

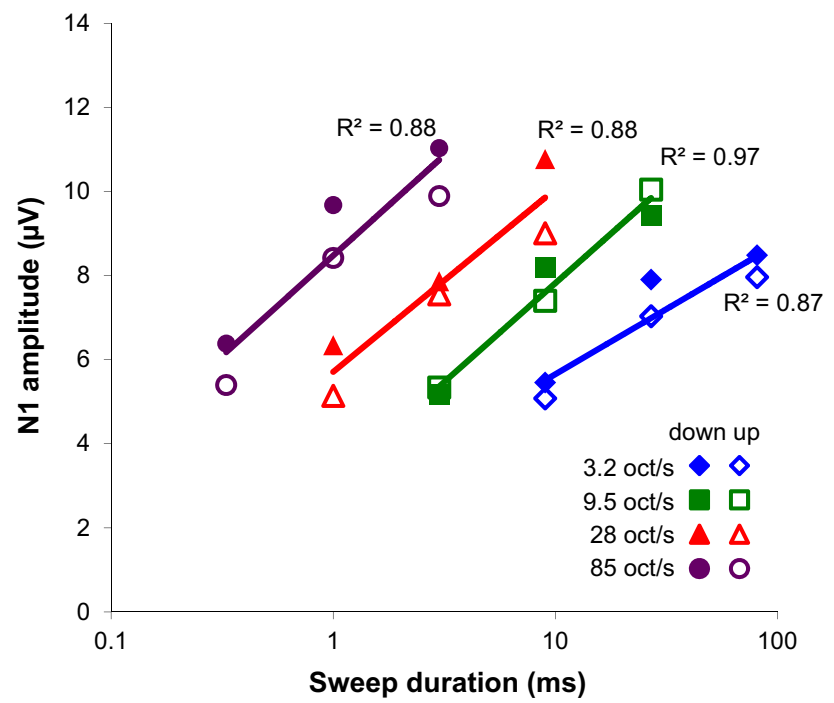

Fig. 4. N1 amplitude as a function of sweep duration, presented for each rate separately and averaged across all 12 subjects. The purple line indicates the fastest sweeps of $85 \mathrm{oct} / \mathrm{s}$, the red line indicates $28 \mathrm{oct} / \mathrm{s}$, the green line $9.5 \mathrm{oct} / \mathrm{s}$, and the blue line $3.2 \mathrm{oct} / \mathrm{s}$. Downward sweeps are indicated with solid symbols and upward sweeps with open symbols. The increase of $\mathrm{N} 1$ amplitude $(A)$, with frequency change $(\Delta f)$, can be described as follows: $A \sim b \log (\Delta f)$. The slope $b$ for a rate of 85 oct $/ \mathrm{s}$ is 4.8 , for 28 oct $/ \mathrm{s} 4.3$, for $9.5 \mathrm{oct} / \mathrm{s}$ 4.7 , and for $3.2 \mathrm{oct} / \mathrm{s} 3.1$

104-132 ms) compared to upward changes (range $102-145 \mathrm{~ms}$ ) (rm ANOVA; $F(1,11)=88.9, p<0.0001$ ). Note that the increase of latency with decrease of rate implies a longer latency with a longer sweep duration for the same frequency step magnitude.

The P2 peak latency followed the $\mathrm{N} 1$ peak by approximately 80-90 ms for each frequency step and rate. To investigate whether the $\mathrm{P} 2$ latency was influenced by the stimulus parameters in a different way than the N1 latency, the latency difference between P2 and N1 was analyzed. This latency difference was not significantly affected by magnitude, rate, or direction of the frequency change ( $\mathrm{rm}$ ANOVA; $p>0.2$ ).

\section{Direction Preference}

Since both the amplitude and the latency of the N1 peak depended on the direction of the frequency change, as indicated by the rm ANOVA statistics; the direction preference was further analyzed for amplitude (Fig. 6a) and latency (Fig. 6b). The direction selectivity as computed for amplitude (DSI) was around 0 for the smaller magnitudes of change; a downward preference appeared for the largest magnitude applied, in particular for the faster sweeps. For individual stimulus conditions, downward preferences were not significant (one-sample $t$ test, Bonferroni corrected, $p>0.05)$. 


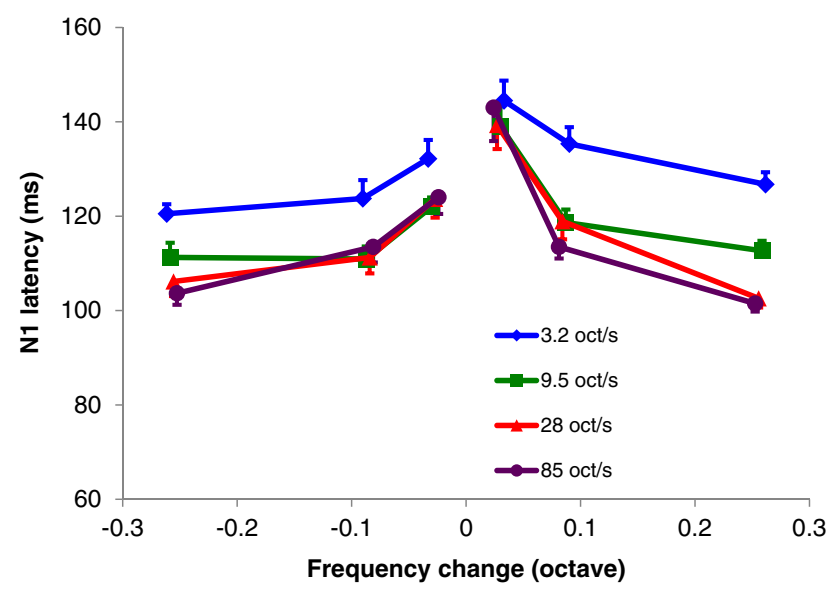

Fig. 5. Average N1 latency as a function of magnitude of the frequency change $(n=12)$. Downward frequency changes are presented on the left (indicated with negative values) and upward changes on the right. Purple lines indicate the sweeps of $85 \mathrm{oct} / \mathrm{s}$, red lines of $28 \mathrm{oct} / \mathrm{s}$, green lines of $9.5 \mathrm{oct} / \mathrm{s}$, and blue lines of $3.2 \mathrm{oct} / \mathrm{s}$. Error bars indicate standard errors of the mean

For the N1 latency, there is a downward preference for most conditions (Fig. 6b), also reflected by Fig. 5 . Where DSI is larger for larger magnitudes, the latency difference is larger for the smaller frequency steps. The smallest frequency change of 0.028 oct displayed a significant downward preference for the higher rates (paired samples $t$ test, Bonferroni corrected $9.5 \mathrm{oct} / \mathrm{s} t(11)=5.36, p=0.002 ; 28 \mathrm{oct} / \mathrm{s} t(11)=4.05$, $p=0.024 ; 85$ oct $/ \mathrm{s} t(11)=4.91, p=0.006)$. Significantly shorter latencies for downward changes were found at 0.086 oct and $9.5 \mathrm{oct} / \mathrm{s}(t(11)=4.16, p=0.024)$ and at 0.257 oct and $3.2 \mathrm{oct} / \mathrm{s}(t(11)=3.69, p=0.047)$.

\section{DISCUSSION}

This study's objective was to investigate the effect of magnitude, rate, and direction of frequency changes on ACCs evoked in normal-hearing human subjects. Our results show that both amplitude and latency of the N1 peak are strongly affected by magnitude, rate, and direction of the frequency change, i.e., larger and earlier N1 peaks can be evoked by increasing the magnitude and/or rate of the frequency change and with downward changes. The P2 component showed a similar effect although only its amplitude was influenced by magnitude and, to a lesser extent, rate of the frequency change while direction had no effect on this peak. Recent literature has aimed at correlations of ACC measures with psychophysical outcomes in order to develop a clinical tool. It is therefore important to know, and relevant for researchers investigating the ACC, that the ACC is affected not only by magnitude but also, secondarily, by rate and direction.
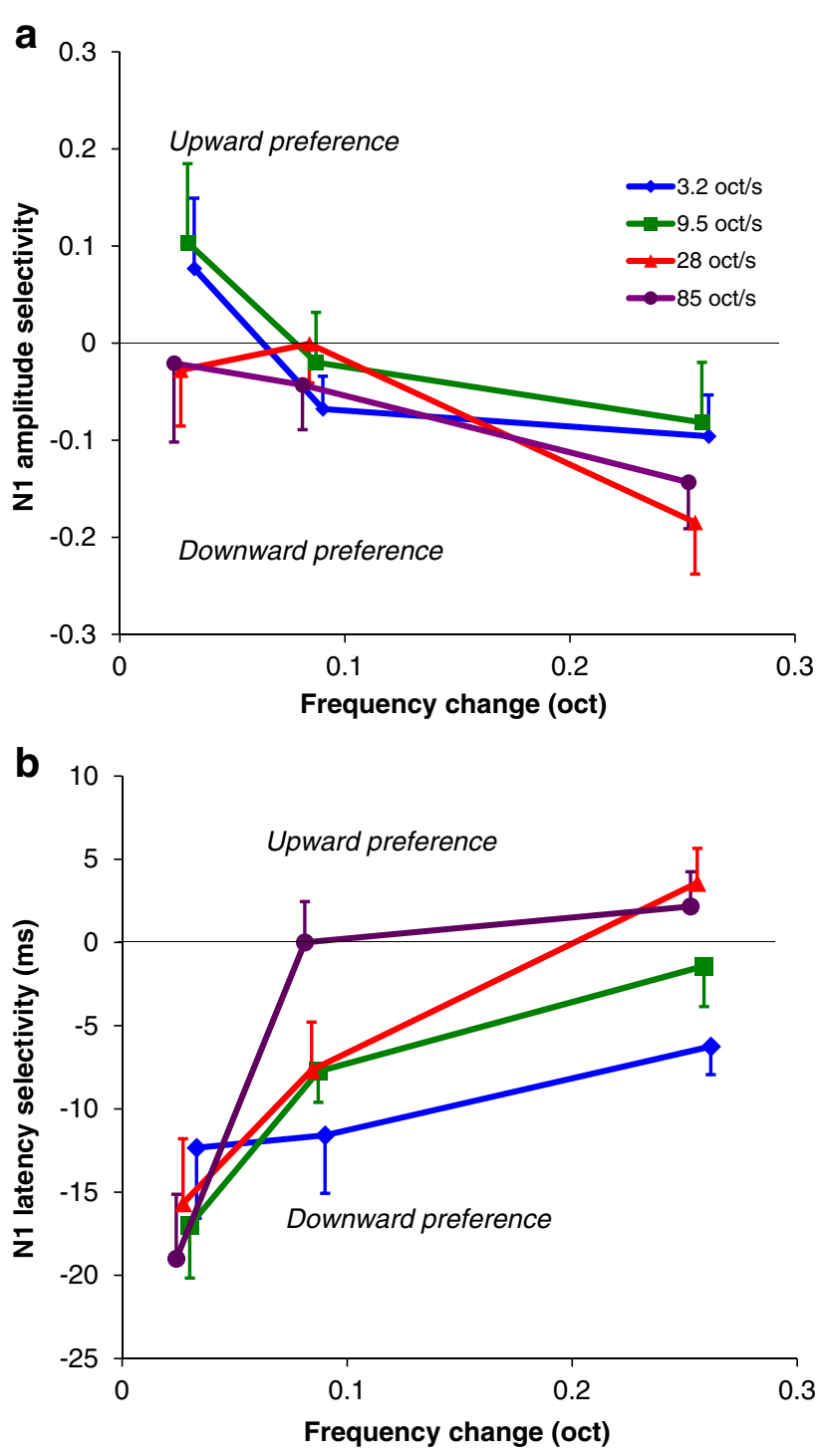

Fig. 6. a N1 amplitude direction selectivity, expressed by DSI, as a function of frequency change $(n=12)$. b N1 latency direction selectivity, expressed by latency difference, as a function of frequency change $(n=12)$. Positive values represent an upward preference (larger amplitude or shorter latency) and negative values a downward preference. Purple lines indicate the sweeps of 85 oct/s, red lines of $28 \mathrm{oct} / \mathrm{s}$, green lines of $9.5 \mathrm{oct} / \mathrm{s}$, and blue lines of $3.2 \mathrm{oct} / \mathrm{s}$. Error bars indicate standard errors of the mean. Random jitter has been added to avoid overlapping data points

Effects of Magnitude and Rate of the Frequency Change on ACC Amplitude and Latency

The increase of ACC N1 and P2 amplitudes and the decrease of N1 latency with increase of frequency change are in line with earlier studies (McCandless and Rose 1970; Martin and Boothroyd 2000; Harris et al. 2008; Pratt et al. 2009; He et al. 2012). Harris et al. (2008) and $\mathrm{He}$ et al. (2012) found that a frequency change of around $1 \%$ of the base frequency was required to evoke a discernable cortical response in young normal-hearing volunteers. In line 
with their results, all subjects in our study displayed a clear response with the smallest frequency change of 0.028 oct which corresponds with approximately $2 \%$.

In addition to the effect of magnitude, our results demonstrated that frequency change rate has a positive effect on N1 amplitude and, to a lesser extent, on P2 amplitude. In correspondence with the effect on amplitude, we found shorter N1 latencies with higher velocities.

This illustrates that the evoked cortical response is dependent on both frequency magnitude and rate, which can be explained as follows. The frequency change consists of an FM sweep and the target tone. Thus, the evoked response consists of the sum of the responses to the FM sweep and to the target tone. An individual cortical neuron may be sensitive to both components or to either of the two (Heil et al. 1992; Shamma et al. 1993; Kowalski et al. 1995; Nelken and Versnel 2000). In primary areas, the response to a pure tone is relatively strong; in nonprimary areas, the response to the FM sweep is relatively strong (Tian and Rauschecker 2004). Cortical neurons respond to the pure tone and FM sweep as long as these fall within the excitatory frequency response area. The number of neurons responding to the FM sweep increases with increase of the sweep magnitude, since the sweep passes through more excitatory frequency areas. Consequently, the evoked response increases with magnitude as indeed we found. The response of an individual neuron to the FM sweep may depend on direction and rate (Heil et al. 1992; Kowalski et al. 1995; Nelken and Versnel 2000; Tian and Rauschecker 2004; Trujillo et al. 2011), while the response to the pure-tone component will not depend on those parameters. Direction and rate selectivities vary widely across the auditory cortical areas; thus, some neurons respond to up sweeps rather than to down sweeps, and some neurons respond to slow sweeps rather than to fast sweeps. The summed evoked response to the FM sweep may actually not vary greatly with direction or rate if neurons with direction and rate selectivities are symmetrically and uniformly distributed. However, dependence on rate can be explained as follows. First, with faster sweeps, the neurons responding to the sweep respond more synchronously. Second, in case of short sweeps, the response to the target tone (the 300-ms tone following the FM sweep, as described in "Methods") will follow the response to the FM sweep quite shortly (within $10 \mathrm{~ms}$ ). This results in a larger total evoked response, whereas in case of a long sweep (in particular $81 \mathrm{~ms}$; see Fig. 1), the responses to FM sweep and target tone will not overlap. Indeed, at the largest step, the responses were substantially smaller for the slowest sweep (where the sweep was longest) than for the other sweeps (Fig. 3). The decrease of latency with increase of rate can be further explained by assuming the response reaches its maximum during the sweep. For instance, considering the ACCs with a decrease of rate for the largest frequency step ( 0.26 oct), we see an increase of latency of about $20 \mathrm{~ms}$ from fastest to slowest sweep while the duration of the sweep increases by $78 \mathrm{~ms}$ (81-3 ms), suggesting the response peaks after a quarter of the sweep. For shorter steps, the response may peak towards the end of the sweep (latency increase of $8 \mathrm{~ms}$ while sweep increases by $9 \mathrm{~ms}$ ).

\section{Comparison to ACCs Reported in Literature}

Compared to the current study, Dimitrijevic et al. (2008), Harris et al. (2008), and He et al. (2012) all reported considerably smaller N1-P2 amplitudes varying between 2.5 and $5 \mu \mathrm{V}$ in response to frequency increases (magnitudes varying 8 to $50 \%$ ) within pure tones. The largest frequency change magnitude in our study, which is 0.257 oct (approximately $20 \%$ ), generated considerable larger N1 amplitudes between 8.5 and $10.7 \mu \mathrm{V}$. This difference in amplitude between studies could occur due to use of different stimuli. Our results demonstrate that cortical potential recordings in response to frequency changes are highly stimulus dependent. In the current study, the first stimulus component before the change has a duration of approximately $3 \mathrm{~s}$. This duration was based on our pilot data obtained in 3 subjects, which indicated that prolonging this component duration from 1 to $3 \mathrm{~s}$ generates approximately 30-50\% larger amplitudes. The stimuli used by Dimitrijevic et al. (2008) and He et al. (2012) had a duration of respectively $400 \mathrm{~ms}$ and $1300 \mathrm{~ms}$ before the change occurred. The enhanced neural responsiveness with a longer stimulus duration before the change might be facilitated due to increased firing ability of separate neurons. Wang et al. (2005) demonstrated that activity of single neurons within the monkey auditory cortex decreases with time during presentation of a 5000-ms constant tone, in particular in response to nonpreferred frequencies. A longer duration of the pure tone before the change could therefore facilitate low activity and thus a generally increased ability to respond to a new stimulus. Harris et al. (2008) reported an $\mathrm{N} 1$ amplitude of only $2.5 \mu \mathrm{V}$ in response to an $8 \%$ frequency increase, which is much smaller than we observed (Figs. 3 and 4). The duration of the reference tone was similar in both studies $(\sim 3 \mathrm{~s})$; however, Harris et al. (2008) presented continuous stimuli, whereas we applied silent intervals of $200 \mathrm{~ms}$ allowing dishabituation and more neural recovery (Martin et al. 2007; Näätänen and Picton 1987), which might explain the larger amplitudes in the current study. 
The N1 latencies we found (between 102 and $145 \mathrm{~ms}$ ) are similar to values reported in the literature, including the strong dependence on magnitude of the change. Harris et al. (2008) reported a latency of approximately $132 \mathrm{~ms}$ in response to an $8 \%$ frequency increase, He et al. (2012) reported an N1 latency of $110 \mathrm{~ms}$ in response to $20 \%$ frequency changes, and Dimitrijevic et al. (2008) reported latencies of approximately $105 \mathrm{~ms}$ in response to a $50 \%$ frequency increase. Indeed, these studies together showed consistently shorter latencies with larger changes. These studies did not report the change rate of their stimuli; therefore, minor differences between studies might occur due to the effects of both rate and varying durations of the first stimulus component. Brown et al. (2015) recorded ACCs in normal hearing subjects in response to changing vowel sounds with the second formant shifting an octave at $400 \mathrm{~ms}$ after onset. This study also reported amplitudes (N1-P2 amplitude $7 \mu \mathrm{V}$ ) and latencies (N1 latency approximately $120 \mathrm{~ms}$ ) which agree with our values considering on the one hand the large change and on the other hand the short duration of the first component.

\section{Direction Preference}

Previous studies using cortical evoked potentials have reported contrasting results with respect to the dependence on frequency change direction (Arlinger et al. 1976; Arlinger and Jerlvall 1979; Maiste and Picton 1989; Pratt et al. 2009). Maiste and Picton (1989) and Pratt et al. (2009) reported larger amplitudes for frequency increases than decreases in contrast to the studies by Arlinger et al. (1976) and Arlinger and Jerlvall (1979) reporting no difference in amplitude between upward or downward sweeps. Brown et al. (2015) used vowel transitions and did not find any differences in ACCs between increases and decreases of the formant frequency. In contrast to these studies, our study demonstrates a downward direction preference for the N1 peak, for the small changes expressed by shorter latencies and for large changes expressed by larger amplitudes. In the current study, we chose to use separate recordings in a randomized order to control for possible interactions between successive stimuli. Moreover, for direction selectivity analyses, we corrected for variance in amplitudes, rather than using absolute peak amplitudes. Inter-subject variance in ACC amplitudes could severely influence calculations of direction selectivity if individual peak amplitudes are averaged.

Taken together, the results of the abovementioned studies indicate that the existence of a general direction preference is weak at most. Various psycho- physical studies, which examined detection ability in human speech and/or spectrotemporal modulations, confirm this notion (Schouten and Pols 1985; Schouten 1986; Dooley and Moore 1988; Chi et al. 1999; Gordon and Poeppel 2002; Luo et al. 2007). It implies that in human auditory cortex up- and downward directions, selective neurons are roughly equally distributed. Preference may be dependent on specific stimulus conditions as suggested by Nelken and Versnel (2000) and as indicated by the current study.

\section{Clinical Implications}

A literature review by Kim (2015) concluded that the ACC as an objective measurement shows reasonable agreement with psychophysical measures and can be reliably recorded in normal-hearing subjects, hearing impaired patients, and cochlear implant users. The ACC might therefore hold clinical value as an objective tool in hearing impairment. When investigating clinical applications, one should be aware the ACC is affected not only by magnitude but also by rate and direction of the change. In order to obtain the clearest response, a large and fast FM sweep is the appropriate stimulus to be used.

\section{ACKNOWLEDGEMENTS}

The authors thank Thijs Dijkgraaf for his technical support.

\section{COMPLIANCE WITH ETHICAL STANDARDS}

Conflict of Interest The authors declare that they have no conflict of interest.

Open Access This article is distributed under the terms of the Creative Commons Attribution 4.0 International License (http://creativecommons.org/licenses/by/4.0/), which permits unrestricted use, distribution, and reproduction in any medium, provided you give appropriate credit to the original author(s) and the source, provide a link to the Creative Commons license, and indicate if changes were made.

\section{REFERENCES}

Amitay S, Irwin A, Hawkey DJ, Cowan JA, Moore DR (2006) A comparison of adaptive procedures for rapid and reliable threshold assessment and training in naive listeners. J Acoust Soc Am 119:1616-1625

Arlinger SD, Jerlvall LB, Ahrén T, Holmgren EC (1976) Slow evoked cortical responses to linear frequency ramps of a continuous pure tone. Acta Physiol Scand 98:412-424 
Arlinger SD, Jerlvall LB (1979) Results of psychoacoustic and cortical evoked potential experiments using frequency and amplitude modulated stimuli. Scand Audiol Suppl 9:229-239

Brown CJ, Jeon EK, Chiou LK, Kirby B, Karsten SA, Turner CW, Abbas PJ (2015) Cortical auditory evoked potentials recorded from nucleus hybrid cochlear implant users. Ear Hear 36:723-732

Brown CJ, Jeon EK, Driscoll V, Mussoi B, Deshpande SB, Gfeller K, AbBas PJ (2017) Effects of long-term musical training on cortical auditory evoked potentials. Ear Hear 38:e74-e84

Chen KH, Small SA (2015) Elicitation of the acoustic change complex to long-duration speech stimuli in four-month-old infants. Int J Otolaryngol, Article ID 562030 2015:1-12

Chi T, GAO Y, GUYTon MC, Ru P, Shamma S (1999) Spectro-temporal modulation transfer functions and speech intelligibility. J Acoust Soc Am 106:2719-2732

Dimitrijevic A, Michalewsi HJ, Zeng FG, Pratt H, Starr A (2008) Frequency changes in a continuous tone: auditory cortical potentials. Clin Neurophysiol 119:2111-2124

Dooley GJ, Moore BC (1988) Duration discrimination of steady and gliding tones: a new method for estimating sensitivity to rate of change. J Acoust Soc Am 84:1332-1337

Dreschler WA, Plomp R (1985) Relations between psychophysical data and speech perception for hearing-impaired subjects. II. J Acoust Soc Am 78:1261-1270

Eggermont JJ, Ponton CW (2002) The neurophysiology of auditory perception: from single units to evoked potentials. Audiol Neurootol 7:71-99

Friesen LM, Tremblay KL (2006) Acoustic change complexes recorded in adult cochlear implant listeners. Ear Hear 27:678685

Gordon M, Poeppel D (2002) Inequality in identification of direction of frequency change (up vs. down) for rapid frequency modulated sweeps. Acoust Res Lett Online 3:29-34

Harris KC, Mills JH, Dubno JR (2007) Electrophysiologic correlates of intensity discrimination in cortical evoked potentials of younger and older adults. Hear Res 228:58-68

Harris KC, Mills JH, He NJ, Dubno JR (2008) Age-related differences in sensitivity to small changes in frequency assessed with cortical evoked potentials. Hear Res 243:47-56

He S, Grose JH, Buchman CA (2012) Auditory discrimination: the relationship between psychophysical and electrophysiological measures. Int J Audiol 51:771-782

Heil P, Rajan R, Irvine DRF (1992) Sensitivity of neurons in cat primary auditory cortex to tones and frequency-modulated stimuli. I. Effects of variation of stimulus parameters. Hear Res 63:108-134

Horst JW (1987) Frequency discrimination of complex signals, frequency selectivity, and speech perception in hearingimpaired subjects. J Acoust Soc Am 82:874-885

Kıм J (2015) Acoustic change complex: clinical implications. J Audiol Otol 19:120-124

Kowalski N, Versnel H, Shamma S (1995) Comparison of responses in the anterior and primary auditory fields of the ferret cortex. J Neurophysiol 73:1513-1523

Luo H, Boemio A, Gordon M, Poeppel D (2007) The perception of FM sweeps by Chinese and English listeners. Hear Res 224:75-83

Martin BA, Boothroyd A (2000) Cortical, auditory, evoked potentials in response to changes of spectrum and amplitude. J Acoust Soc Am 107:2155-2161

Martin BA, Tremblay KL, Korczak P (2008) Speech evoked potentials: from the laboratory to the clinic. Ear Hear 29:285313

Martin BA, Tremblay KL, Stapells DR (2007) Principles and applications of cortical auditory evoked potentials. In: Burkard
RF, Don M, Eggermont JJ (eds) Auditory evoked potentials: basic principles and clinical application. Lippincott Williams and Wilkins, Philadelphia, pp 482-507

Maiste A, Picton T (1989) Human auditory evoked potentials to frequency-modulated tones. Ear Hear 10:153-160

McCandless GA, Rose DE (1970) Evoked cortical responses to stimulus change. J Speech Hear Res 13:624-634

NäÄtänen R, Picton T (1987) The N1 wave of the human electric and magnetic response to sound: a review and an analysis of the component structure. Psychophysiology 24:375-415

Nelken I, Versnel H (2000) Responses to linear and logarithmic frequency-modulated sweeps in ferret primary auditory cortex. Eur J Neurosci 12:549-562

Noordhoek IM, Houtgast T, Festen JM (2001) Relations between intelligibility of narrow-band speech and auditory functions, both in the 1-kHz frequency region. J Acoust Soc Am 109:11971212

Ostroff JM, Martin BA, Boothroyd A (1998) Cortical evoked response to acoustic change within a syllable. Ear Hear 19:290297

Papakonstantinou A, Strelcyk O, Dau T (2011) Relations between perceptual measures of temporal processing, auditory-evoked brainstem responses and speech intelligibility in noise. Hear Res 280:30-37

Pratt H, Starr A, Michalewski HJ, Dimitrijevic A, Bleich N, Mittelman N (2009) Auditory-evoked potentials to frequency increase and decrease of high- and low-frequency tones. Clin Neurophysiol 120:360-373

Presacco A, Middlebrooks JC (2018) Tone-evoked acoustic change complex (ACC) recorded in a sedated animal model. J Assoc Res Otolaryngol 19:451-466

Schouten ME, Pols LC (1985) Identification and discrimination of sweep tones. Percept Psychophys 37:369-376

Schouten ME (1986) Three-way identification of sweep tones. Percept Psychophys 40:359-361

Sek A, Moore BC (1995) Frequency discrimination as a function of frequency, measured in several ways. J Acoust Soc Am 97:24792486

Shamma S, Fleshman J, Wiser R, Versnel H (1993) Organization of response areas in ferret primary auditory cortex. J Neurophysiol 69:367-383

Strelcyk O, Dau T (2009) Relations between frequency selectivity, temporal fine-structure processing, and speech reception in impaired hearing. J Acoust Soc Am 125:3328-3345

Tian B, Rauschecker JP (2004) Processing of frequency-modulated sounds in the lateral auditory belt cortex of the rhesus monkey. J Neurophysiol 92:2993-3013

Tremblay KL, Billings CJ, Friesen LM, Souza PE (2006) Neural representation of amplified speech sounds. Ear Hear 27:93-103

Tremblay KL, Friesen L, Martin BA, Wright R (2003) Test-retest reliability of cortical evoked potentials using naturally produced speech sounds. Ear Hear 24:225-232

Trujlllo M, Measor K, Carrasco MM, Razak KA (2011) Selectivity of frequency-modulated sweeps in the mouse auditory cortex. J Neurophysiol 106:2825-2837

Wang X, Lu T, SNider RK, Liang L (2005) Sustained firing in auditory cortex evoked by preferred stimuli. Nature 435:342-346

Publisher's Note Springer Nature remains neutral with regard to jurisdictional claims in published maps and institutional affiliations. 